\title{
RELATING COVARIANT AND CANONICAL APPROACHES TO TRIANGULATED MODELS OF QUANTUM GRAVITY
}

\author{
MATTHIAS ARNSDORF
}

\begin{abstract}
In this paper explore the relation between covariant and canonical approaches to quantum gravity and $B F$ theory. We will focus on the dynamical triangulation and spin-foam models, which have in common that they can be defined in terms of sums over space-time triangulations. Our aim is to show how we can recover these covariant models from a canonical framework by providing two regularisations of the projector onto the kernel of the Hamiltonian constraint. This link is important for the understanding of the dynamics of quantum gravity. In particular, we will see how in the simplest dynamical triangulations model we can recover the Hamiltonian constraint via our definition of the projector. Our discussion of spin-foam models will show how the elementary spin-network moves in loop quantum gravity, which were originally assumed to describe the Hamiltonian constraint action, are in fact related to the time-evolution generated by the constraint. We also show that the Immirzi parameter is important for the understanding of a continuum limit of the theory.
\end{abstract}

\section{IntRoduCtion}

General relativity can be quantised by canonical or covariant methods depending on whether one regards space or space-time as more fundamental. Because of the incomplete nature of the subject the relation between the two approaches is not entirely clear, even though one expects that in appropriate circumstances they should be equivalent. The broad aim of this paper is to contribute to this discussion.

General relativity is a completely constrained system and hence quantum gravity distinguishes itself from ordinary field theory in that the Hamiltonian vanishes weakly. This implies for a canonical formalism that there is no propagator, since there is no external time variable with respect to which it could be defined. Instead the central object is the generalised projector onto the solution space of the Hamiltonian constraint $\hat{H}(x)$. Formally, this projector can be written as:

$$
\hat{P}=\int[d T(x)] e^{-i \int_{\Sigma} d^{n} x \hat{H}(x) T(x)},
$$

where $[d T(x)]$ is a measure on the space of scalar fields $T(x)$ and $\Sigma$ is a spatial slice of dimension $n$ of the space-time manifold $\mathcal{M}=\Sigma \times \mathbb{R}$. This operator then defines the physical Hilbert space of the theory, from which we have to extract all physical predictions by the construction of suitable observables.

This projector can be expressed as a phase space path integral in an analogous way as is done for the propagator in ordinary field theory. Hence, we expect this projector to be the link between the above canonical description and covariant approaches to the quantisation of gravity.

Date: October 2001. 
Indeed, the starting point for the covariant approach is the expression

$$
Z\left(g_{1}, g_{2}\right):=\int_{g_{1}}^{g_{2}}[d \mathbf{g}] e^{-i S[\mathbf{g}]}
$$

which describes a sum over all space-time geometries $\mathbf{g}$ on the cylinder $\Sigma \times[0,1]$ with boundary geometries $g_{1}$ and $g_{2}$. Two promising approaches towards making sense of this expression are the dynamical triangulation and the spin-foam models. These have in common that they they replace the above sum over geometries by a sum over space-time triangulations.

The aim of this paper will be to show that the projector $\hat{P}$ can be suitably regularised so that it describes a sum over space-time triangulations. More precisely, we will be discussing canonical theories of quantum gravity that have a Hilbert space of kinematical states $\Delta$ given by triangulations or dual graphs of the spatial slice $\Sigma$. We will provide two different regularisations of the projector such that its matrix elements $\left\langle\Delta_{1}|\hat{P}| \Delta_{0}\right\rangle$ are given by a sum over triangulations of the cylinder $\Sigma \times[0,1]$, with boundary triangulations $\Delta_{0}$ and $\Delta_{1}$. The amplitudes of the triangulations will be given by the dynamical triangulation and spin-foam models respectively.

The idea will be to decompose the projector into a product of "small" unitary local proper time evolutions. Acting on an initial triangulation $\Delta$ each elementary evolution corresponds to a gluing of an $(n+1)$-dimensional simplex to the boundary. In this way we build up a sum over space-time triangulations with the correct amplitude.

This link between canonical and covariant theories is important for the understanding of the dynamics of these quantum gravity models and for providing a space-time picture in the canonical framework. Ultimately, one would like to relate a given Hamiltonian to the amplitude of a corresponding covariant model. As we will see this can be achieved in the simplest case of $1+1$ dynamical triangulations. The discussion of spin foam models of quantum gravity and the closely related topological $B F$ theories, will clarify their relation to canonical loop quantum gravity. In particular, we will see that elementary moves of spin-networks, which were thought to describe the action of the Hamiltonian constraint, are in fact related to time-evolutions. A better understanding of this relation should help in deciding which of the current proposals for spin foam models provides a correct description of quantum gravity and also shed light on the nature of the Hamiltonian constraint of loop quantum gravity.

This work builds on a previous attempt to derive a spin foam model from $3+1$ loop quantum gravity by Reisenberger and Rovelli [20], which was developed further by Rovelli in [23]. Our approach will differ in the regularisation of the projector. We will see in this paper how this leads to many attractive features:

- By providing two different regularisations we can describe both the spin-foam and the dynamical triangulation models.

- Our expansions are closely related to the derivation of the path integral in quantum mechanics and field theory.

- They provide a clear role for the $[d T(x)]$ integration over scalar (lapse) functions in the projector and avoid infinities due to this integration.

- They provide a very natural interpretation of the addition of space-time simplices as local proper time evolutions.

- They avoid over counting of space-time triangulations.

- We obtain an understanding of the continuum limit of spin-foam models. 
- Most importantly one can show that in $B F$ theories and $2+1$ loop quantum gravity the addition of tetrahedra, when viewed as an operation on physical states, leaves these invariant. This suggests that these actions should be viewed as evolutions and not actions of the Hamiltonian constraint operator.

- In addition, in 1+1 dynamical triangulation we can show explicitly how we can derive the correct Hamiltonian operator from the covariant model via our expansion of the projector.

We begin our discussion by describing the formal relation between the projector and path integrals for quantum gravity. This will be made concrete in the dynamical triangulations framework focusing on $1+1$ dimensions, where results can be stated most explicitly and simply. We then continue to generalise our results to higher dimensions before moving on to consider spin-foam models and loop quantum gravity.

\section{Canonical vis. Covariant}

In this section we describe the generalised projector and show how it is formally related to a sum over geometries. We will find that canonical and covariant quantum gravity are equivalent up to the choice of range in the integration over lapse functions.

2.1. Canonical quantum gravity. The canonical description of general relativity requires that we restrict our attention to space-time manifolds $\mathcal{M}$ that allow a split into space and time, i.e. manifolds diffeomorphic to $\mathcal{M}=\Sigma \times \mathbb{R}$. Here and in the following $\Sigma$ will denote the $n$-dimensional spatial slice, which we will take to be compact.

General relativity is a completely constrained system, i.e. the Hamiltonian for the theory vanishes weakly. This can be viewed as a consequence of diffeomorphism invariance and the absence of an external time parameter. Hence quantisation follows the Dirac prescription which requires that we quantise the unconstrained classical system and impose the constraints as quantum operators on an initial, kinematical, Hilbert space $\mathcal{H}_{k i n}$. Loosely speaking, the constraints implement the diffeomorphism invariance of the theory. Typically, we distinguish between the "momentum" constraints $H_{i}$, which generate diffeomorphisms of the spatial slice $\Sigma$ and the "Hamiltonian" constraint $H$, which is responsible for diffeomorphisms normal to the spatial slice and is hence associated with dynamics. If connection instead of metric variables are used in the coordinatisation of the phase space there will be additional "Gauss" constraints, enforcing invariance under internal gauge transformations corresponding to the rotation of tetrads.

Physical states lie in the kernel of the constraint operators. If this space is nontrivial and can be endowed with an inner product, we obtain the final physical Hilbert space $\mathcal{H}_{\text {phys }}$. Note the absence of a Hamiltonian operator on $\mathcal{H}_{\text {phys }}$, resulting in the the lack of a notion of evolution or propagator. States are interpreted as entire histories, which makes the extraction of physical information from this frozen picture highly problematic.

These conceptual matters will not concern us. Instead we need to face the following technical issue related to our problem of constructing a path integral for general relativity. Whenever 0 lies in the continuous part of the spectrum of one of the constraint operators, the corresponding eigenvectors will not be normalisable. Hence the construction of the physical Hilbert space is non-trivial and proceeds by 
the so-called refined algebraic quantisation (RAQ) procedure (see [15 for a review and references). The idea is to look for distributional solutions to the constraints, i.e. functionals on the kinematical state space 1 . The solutions are precisely those functionals that are constant on the orbits of the unitary action generated by the constraints. Equivalently we could be view the orbits themselves as the generalised solutions. The key step is to define an inner product on these physical states, which is done by introducing a generalised projector $P$. This is a map from $\mathcal{H}_{\text {kin }}$ onto the space of functionals, which maps a vector to its orbit under the constraint action. The image of the map $P$ will be referred to as the solution space of the constraints.

Let us denote physical states by a tilde, i.e. $\tilde{\psi}:=P \psi, \tilde{\phi}:=P \phi$ with $\psi, \phi \in \mathcal{H}_{k i n}$, then the physical inner product is given by:

$$
\langle\tilde{\psi}, \tilde{\phi}\rangle_{p h y s}:=\tilde{\phi}[\psi]
$$

where the right hand side denotes the action of the functional $\tilde{\phi}$ on the kinematical state $\psi$. In many cases $P$ can be constructed by the method of group averaging. In this case we have:

$$
\langle\psi, \phi\rangle_{p h y s}:=\int d g\langle\psi, \hat{U}(g) \phi\rangle_{k i n}:=\langle\psi, \hat{P} \phi\rangle_{k i n}
$$

where $\hat{U}(g)$ denotes the group action generated by the constraint under consideration and $d g$ is a suitable measure on the group. We have also made use of the kinematical inner product $\langle\cdot, \cdot\rangle_{\text {kin }}$ on $\mathcal{H}_{\text {kin }}$.

Completion of the solution space with respect to the physical inner product gives us the physical Hilbert space $\mathcal{H}_{\text {phys }}$. The operator $\hat{P}$ defined above is called the generalised projection operator since it reduces to an ordinary projection onto the kernel of the constraints if the corresponding group action is compact. But the power of the approach lies in the fact that it can be applied in other cases also, in fact whenever the above integral converges, group averaging defines the unique generalised projector [8].

When applied to the momentum and Gauss constraints the above procedure is implemented simply, by demanding that states are invariant under the action of spatial diffeomorphisms and gauge transformations respectively. The Hamiltonian constraint, however, causes difficulties since it generates diffeomorphisms which are normal to the spatial slice and hence does not have a simple geometrical action on kinematical states. Indeed, the Hamiltonian constraint is related to evolution in coordinate time hence we expect the projector corresponding to $H$ to be related to the propagator in field theory. This is what we explore in the following.

In analogy to the group averaging procedure we can write down a formal expression for the generalised projection operator corresponding to the Hamiltonian constraint operator $\hat{H}(x)$ :

$$
\hat{P}=\int[d T(x)] e^{-i \hat{H}[T]}
$$

where $\hat{H}[T]:=\int_{\Sigma} d^{n} x \hat{H}(x) T(x)$. Here $[d T(x)]$ denotes a measure on the space of scalar functions $T(x)$, which we leave unspecified. The integral should be understood as an integral over the generators of the algebra generated by $\hat{H}(x)$. Since the exponential $\exp (-i \hat{H}[T])$ is linear in $T(x)$, the above can also be viewed as a

\footnotetext{
${ }^{1}$ To be precise we need to consider a dense subspace of the kinematical Hilbert space. This technical issue will not be important for our formal discussion.
} 
expression of a product of delta functions imposing the constraint $\prod_{x \in \Sigma} \delta[\hat{H}(x)]$. Alternatively, we can understand the above in the following way. The expression $\left\langle\psi, e^{i \hat{H}[T]} \phi\right\rangle_{\text {kin }}$ defines the "propagator" in multi-fingered proper time $T(x)$ between two kinematical states $\psi$ and $\phi$, which describes geometries on the boundaries of the cylinder $[0,1] \times \Sigma$. Since physical results should be independent of the arbitrary proper time separation between initial and final splices, we should integrate over all possible $T(x)$. This then defines the above projector. A more in depth discussion is given in [20]. We will now show in more detail how the projector and the path integral for quantum gravity are related.

2.2. Covariant quantum gravity. The projector can be expressed as a phase space path integral as is demonstrated by Klauder [12] for general constrained systems. This phase space integral can also be derived from a sum over geometries approach to quantum gravity, as has been investigated in detail by Teitelboim [29, 31], forging the link between covariant and canonical frameworks.

Let us denote the points in the phase space of general relativity as $[p(x), q(x)]$, $x \in \Sigma$ and let us furthermore assume we have eigenstates $|q\rangle \in \mathcal{H}_{k i n}$ of the corresponding configuration operators. We can then express the physical inner product:

$$
\left\langle\tilde{q}_{1}, \tilde{q}_{0}\right\rangle_{\text {phys }}:=\int[d T(x)]\left\langle q_{1}\left|e^{-i \hat{H}[T]}\right| q_{0}\right\rangle
$$

as a path integral in complete analogy with the derivation of the path integral in elementary quantum mechanics. More concretely, we imagine that the operator $\exp (-i \hat{H}[T])$ is evolving between a state describing an initial geometry at coordinate time 0 and a final geometry at coordinate time 1 . We then write:

$$
e^{-i \hat{H}[T]}=\lim _{\epsilon \rightarrow 0} \prod_{k=1}^{N} e^{-i \epsilon \hat{H}[T]},
$$

with $\epsilon=1 / N$. Then using the standard techniques we obtain formally:

$$
\left\langle\tilde{q}_{1}, \tilde{q}_{0}\right\rangle_{p h y s}=\int[d T(x)] \int_{q_{0}}^{q_{1}}[D p][D q] e^{i \int_{0}^{1} d t \int d^{n} x\left(p(x)_{t} \dot{q}(x)_{t}-H(p, q) T(x)\right)}
$$

The expression on the right is an integral over all paths in phase space

$$
t \rightarrow\left[q(x)_{t}, p(x)_{t}\right] \quad t \in[0,1]
$$

with fixed initial and final configurations $q_{0}$ and $q_{1}$. The measure $[D q]:=\prod_{t=0}^{1}[d q(x)]$ is a product of suitably normalised measures $[d q(x)]$ on the space of functions $q(x)$ with a similar expression for $[D p]$.

This is the expression that is obtained if we start from a sum over histories approach to quantum gravity modulo ambiguities concerning the integration range in the $[d T]$ integral. There one is interested in defining the quantity:

$$
\int_{g_{0}}^{g_{1}}[d \mathbf{g}] e^{-i S[\mathbf{g}]}
$$

where $S$ denotes the action for general relativity. The integral is over all space-time geometries $2 \mathrm{~g}$ that interpolate between initial and final geometries $g_{0}, g_{1}$ on the boundary of the cylinder $[0,1] \times \Sigma$. By a geometry we mean an equivalence classes of metrics under diffeomorphisms.

\footnotetext{
${ }^{2}$ Here and in the following bold face characters will correspond to space-time quantities
} 
This sum over geometries can be reexpressed as a phase space path integral. In metric variables the phase space coordinates are given by the metrics $g_{i k}(x)$ on the slice $\Sigma$ and their conjugate momenta $p^{i k}(x)$.

By choosing a foliation of the space-time cylinder we can rewrite the gravitational action in Hamiltonian form. The action is then a function of the paths $t \rightarrow\left[g_{i k}(x, t), p^{i k}(x, t)\right]$, where the pair $\left[g_{i k}(x, t), p^{i k}(x, t)\right]$ describes the geometry of the spatial slice $\{t\} \times \Sigma$

$$
S\left[N, N^{i}, p, g\right]=\int_{0}^{1} d t \int_{\Sigma} d^{n} x\left(p^{i k} \dot{g}_{i k}-N(x, t) H\left(g_{i k}, p^{i k}\right)-N^{i}(x, t) H_{i}\left(g_{i k}, p^{i k}\right)\right)
$$

where $N$ and $N_{i}$ are the lapse and shift functions which encode the information on how the foliation of space-time is chosen.

The constraints generate gauge transformations that relate physically indistinguishable variables. Hence, to avoid multiple counting of equivalent paths in the integral (8) we need to fix a gauge. One of the simplest possible gauge choices, as described by Teitelboim [31], is the proper time gauge $\dot{N}=0, N^{i}=0$. These conditions fix the available freedom up to diffeomorphisms of the initial or final slices. Hence one can rewrite the integral (8) as an integral over paths in phase space with initial and final geometries $g(x, 0)$ and $g(x, 1)$ kept fixed:

$$
\int[d T(x)] \prod_{t=0}^{1}\left[d p^{i k}(x, t)\right]\left[d g_{i k}(x, t)\right] e^{i \int_{0}^{1} d t \int_{\Sigma} d^{n} x\left(p^{i k} \dot{g}_{i k}-T(x) H\left(g_{i k}, p^{i k}\right)\right)}
$$

where the integral is over the functions $T(x):=N(x, t)$, which give the multifingered proper time separation between initial and final spatial slices. This integral has the same form as the one derived from the canonical projector in equation (6). Note however, that the integration measures need to be chosen in such a way that the above integral becomes independent on our choice of gauge, otherwise one needs to include ghosts terms in the above action as described by Teitelboim [29]. Alternatively, by choosing the appropriate measures Klauder has derived the above directly, without the prior imposition of the gauge conditions [12]. We will not be concerned with these issues as the measures will be implicitly determined by the choice of models considered later, which provide concrete realisations of the formal path-integral expressions given in this section.

2.3. Relating canonical and covariant approaches. We are interested in the range of integration of the $[d T(x)]$ integral which we have left unspecified and which is responsible for a subtlety in the relation between canonical and covariant approaches. First we note that in order for the lapse function to arise from a well defined foliation it is necessary that $T(x) \neq 0$ for all $x$. Since $T(x)$ is a continuous function this implies that $T(x)$ is either entirely positive or entirely negative.

If the integral (10) is to project onto the kernel of the Hamiltonian constraint and be equivalent to the expression (6) then we need integrate over both positive and negative lapse functions, as is clear from the analogy between the projector and a product of delta functions.

However, if we want the phase space integral (10) to be equivalent to the sum over geometries integral (8), then we should only integrate over half of the allowed $T(x)$, say only those for which $T(x)>0$. To see this we recall that that the function $T(x)$ encodes how the space-time manifold $\mathcal{M}$ is foliated as $[0,1] \times \Sigma$. In equation (10) we 
are integrating over the variables $\left[T(x), g_{i k}, p^{i k}\right]$. The function $T(x)$ determines how the 3 -metric on each slice $\{t\} \times \Sigma$ and their conjugate momenta are glued together to give a space-time metric on $\mathcal{M}$. However, a change in sign of the proper time $T(x)$ simply corresponds to reversing the foliation of $\mathcal{M}$ and hence $\left[T(x), g_{i k}, p^{i k}\right]$ and $\left[-T(x), g_{i k}, p^{i k}\right]$ will describe equivalent space-time metrics.

This symmetry is not enforced by the constraints as they only identify configurations that can be related by infinitesimal deformations. Hence the sum over geometries expression is not exactly equivalent to the phase space integral derived from the canonical picture due to a larger symmetry group in the sum over geometries case. This is reflected in the range of integration in the integral 3 (10).

This feature can be seen clearly in the discussion of the relativistic particle, which can be viewed as general relativity in $0+1$ dimensions. If we choose the half infinite range of integration then the phase space path integral will define the Feynman propagator (an in depth discussion of the different Greens functions that can be obtained in this approach is given by Halliwell and Ortiz in 10]). This is the same result that is obtained if we try to define the sum over geometries integral, which is just a function of the length of the particle trajectory. One approach to do this is to use a dynamical triangulations approach in the Euclidean regime and then Wick rotate the result, which gives us precisely the Feynman propagator.

Let us summarise. We have seen that $\hat{P}$ defined in equation (3) is an important operator for canonical quantum gravity. It has two interpretations depending on the $T(x)$ integration range:

1. For the full integration range we obtain the projector onto the kernel of the Hamiltonian constraint;

2. For the half-infinite integration range $\hat{P}$ is related to the sum over geometries. In either case $\hat{P}$ can be described by a path integral.

In the following we will show how this can be made concrete by discussing first the dynamical triangulations and second the spin-foam model of quantum gravity. The former gives a definition of a sum over geometries whereas the latter is a path-integral definition of the projector. We will see how these descriptions can be recovered from two respective regularisations of $\hat{P}$ defined on the appropriate kinematical Hilbert spaces.

\section{DynAmical TRIANgUlations}

The dynamical triangulations approach to quantum gravity attempts to regularise and define the path integral given by equation (8) (c.f. [1] for details and references). The idea is to work with manifestly diffeomorphism invariant quantities and thus avoid difficulties concerning gauge fixing. This is achieved by replacing the smooth space-time metric manifold by a simplicial manifold constructed from equilateral $(\mathrm{n}+1)$-dimensional simplices of geodesic edge length $a$. Here and in the following we will be working with Riemannian metrics. A Lorentzian framework for dynamical triangulations exists [2] but for our purposes the Riemannian case will suffice to exemplify all the features of our construction.

We are approximating the sum over geometries by a sum over triangulations of the space-time manifold. The weight of each triangulation is given by the Euclidean

\footnotetext{
${ }^{3}$ Teitelboim gives an argument why one might want to restrict the range of integration in the canonical case also 30. The idea is that one should implement causality by demanding that one integrates only over configurations where the final slice is to the future of the initial one.
} 
Regge action, which can be expressed solely as a function of the squared edge lengths. But note that in contrast to Regge calculus we are keeping the edge lengths fixed - triangulations differ only by the connectivity of the simplices. The challenge is to find a non-trivial continuum limit of this statistical model by taking $a \rightarrow 0$, and appropriately rescaling the bare Newton and cosmological constants. This works well in $1+1$ dimensions, where one can recover results from standard Liouville theory. In higher dimensions evidence for a interesting continuum limit is still lacking.

Our goal is to show how the sum over triangulations can be recovered from a canonical theory via an expansion of the projector defined in equation (3). We assume again that space-time is of the form $\mathcal{M}=\Sigma \times \mathbb{R}$. The kinematical Hilbert $\mathcal{H}_{k i n}$ space for our theory is constructed from the free vector space generated by all equilateral triangulations $\Delta$ of $\Sigma$. By identifying the edges as geodesics of length $a$ we can interpret states as piecewise-linear geometries.

We will distinguish between auxiliary and kinematical states. Auxiliary states are actual embedded triangulations of $\Sigma$, whereas kinematical states are equivalence classes of auxiliary states under diffeomorphisms, i.e. we can think of them as non-embedded or abstract triangulations which are simplicial manifolds with the topology of $\Sigma$.

An inner product is specified on the space of triangulations by demanding that inequivalent triangulations are orthogonal i.e.:

$$
\left\langle\Delta, \Delta^{\prime}\right\rangle:=\delta_{\Delta, \Delta^{\prime}}
$$

Completion of the space of kinematical triangulations with respect to this inner product gives us $\mathcal{H}_{k i n}$.

We are interested in the projector $\hat{P}$ acting on this space. From the discussion in the last section we know that $\hat{P}$ is related to the sum over geometries, which in the dynamical triangulation approach is given by

$$
Z_{a}\left(\triangle_{1}, \triangle_{0}\right):=\sum_{\mathbf{\Lambda}} e^{-S[\mathbf{\Lambda}]}
$$

which denotes the sum over all space-time triangulations $\boldsymbol{\Delta}$ with boundaries $\Delta_{0}$ and $\triangle_{1}$ weighted by the exponential of the Regge action $S[\mathbf{\Lambda}]$. In particular, we expect that by restricting the integration range in the projector we should have:

$$
\int[d T(x)]_{+}\left\langle\Delta_{1}\left|e^{-\hat{H}_{a}[T]}\right| \triangle_{0}\right\rangle=Z_{a}\left(\Delta_{1}, \triangle_{0}\right)
$$

where $[d T(x)]_{+}$denotes an integration over positive proper-time functions only Note that because we are doing a Riemannian calculation we are using real amplitudes.

We now show how this can be demonstrated by choosing a suitable regularisation of the projector

\footnotetext{
${ }^{4}$ Note that this restriction implies that the expression on the left of equation 13 does not define the physical inner product.
} 
3.1. Regularisation of the projector I. Let us consider the projector with halfinfinite integration range

$$
\begin{aligned}
\hat{P} & =\int[d T(x)]_{+} e^{-\int d^{n} x T(x) \hat{H}(x)} \\
& =\int_{0}^{\infty} d v \int[d T(x)]_{+} \delta\left[\int d^{n} x T(x)=v\right] e^{-\int d^{n} x T(x) \hat{H}(x)} .
\end{aligned}
$$

Note that the dimension of $v$ is naturally that of space-time volume. Hence we are splitting the sum over all geometries into a sum over geometries with fixed volume and then summing over all possible volumes.

To regularise the functional integral, we allow the function $T(x)$ to only take values in discrete steps of $\epsilon$. In other words $T(x)=t(x) \epsilon$ with $t(x) \in \mathbb{N}$. We also write the spatial integrals as Riemann sums. To do this we assume that we have given some metric on $\Sigma$ with respect to which we can define a square lattice with lattice spacing $l$. We will denote the set of vertices of this lattice by $V$. This gives us a regulated projector $\hat{P}_{l, \epsilon}$.

$$
\begin{aligned}
\hat{P}_{l, \epsilon} & =\int_{0}^{\infty} d v \int[d T]_{\epsilon} \delta\left[l^{n} \epsilon \sum_{x \in V} t(x)=v\right] e^{-\sum_{x \in V} \epsilon l^{n} t(x) \hat{H}(x)} \\
& =\sum_{s=0}^{\infty} \int[d T]_{\epsilon} \delta\left[\sum_{x \in V} t(x)=s\right] \prod_{x \in V} \prod_{k=0}^{t(x)} e^{-\epsilon l^{n} \hat{H}(x)}
\end{aligned}
$$

where $[d T]_{\epsilon}$ denotes a measure on the positive discretised lapse functions.

Let us set

$$
\hat{U}_{l, \epsilon}(x):=e^{-\epsilon l^{n} \hat{H}(x)} .
$$

One can see that the above delta function imposes the condition that the product $\prod_{x \in V} \prod_{k=0}^{t(x)} \hat{U}_{l, \epsilon}(x)$ has exactly $s$ factors. The integral over the discretised lapse function then ensures that for fixed $s$ we sum over all possible $t(x)$ satisfying the constraint. More precisely we have:

$$
\hat{P}_{l, \epsilon}=\sum_{s=0}^{\infty} \sum_{\vec{x} \in V^{s}} \hat{U}_{l, \epsilon}\left(x_{1}\right) \cdots \hat{U}_{l, \epsilon}\left(x_{s}\right)
$$

where the second sum is over all possible ordered $s$-tuples $\vec{x}:=\left(x_{1}, \ldots, x_{s}\right) \in V^{s}$. The ordering arises in the replacement of the spatial integrals by Riemann sums. For example, in $(1+1) \mathrm{d}$ the ordering implies that $x_{1} \leq x_{2} \leq \ldots \leq x_{s}$.

The continuum projector is recovered in the limits $\epsilon, l \rightarrow 0$, whereby we need to check that the results are independent of the lattice chosen in the regularisation.

In the following we will see that this expansion allows a very natural interpretation. The operator $\hat{U}_{l, \epsilon}(x)$ will correspond to a local evolution of a state in proper time $\epsilon$, which will be identified with the addition of a space-time simplex to the boundary triangulation. In this way the above sum will correspond to a sum over triangulations of space-time with the number of tetrahedra in a particular triangulation given by $s$. In the next sections we will discuss this in detail in the context of $1+1$ dimensions.

\footnotetext{
${ }^{5}$ Although this is strictly speaking not a projector we will, by abuse of notation, continue to refer to it as such.
} 


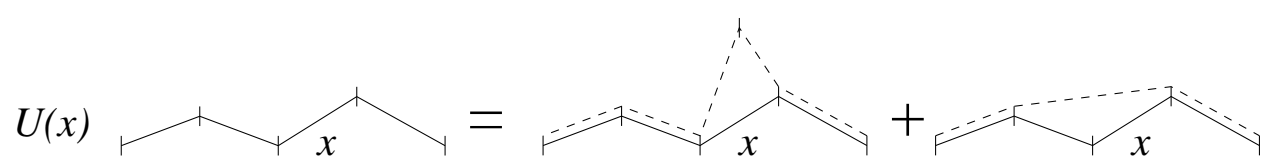

FiguRE 1. The evolution operator acts on a kinematical state by a adding a triangle in two possible ways. This creates a new kinematical state which is depicted by the dashed line.

3.2. $\mathbf{1}+\mathbf{1}$ dimensions. In $1+1$ dimensions the action for general relativity is proportional to the volume of space-time: $S[\mathbf{g}]=\lambda \int_{\mathcal{M}} \sqrt{-\operatorname{det} \mathbf{g}}$, where $\lambda$ denotes the cosmological constant. In the following we will be approximating the sum over geometries by a sum over triangulations of the cylinder $S^{1} \times[0,1]$.

Space-time simplices are simply triangles with area proportional to $a^{2}$. Hence the transition amplitude between two boundary states $\Delta_{0}$ and $\Delta_{1}$ is:

$$
Z_{a}\left(\triangle_{1}, \triangle_{0}\right)=\sum_{\mathbf{\Delta}} e^{-\lambda a^{2} N_{\Delta}}
$$

where the sum is over space-time triangulations $\boldsymbol{\Delta}$ with the appropriate boundaries, and $N_{\mathbf{\Delta}}$ is the number of triangles in $\mathbf{\Lambda}$.

Auxiliary states correspond to triangulations of $S^{1}$ and are thus an embedded sequence of links. They can be characterised by the coordinates $v_{i}$ of the vertices between the links, i.e. they are of the form $|\vec{v}\rangle:=\left|v_{1} \ldots v_{n}\right\rangle$, where $n$ is the number of links. Since the only diffeomorphism invariant information contained in the auxiliary states is the number of links, kinematical states can be though of an abstract chain of links denoted by $|n\rangle$. These are the sates spanning $\mathcal{H}_{k i n}$.

The aim is to find a to find a definition of $\hat{U}_{l, \epsilon}(x)$ acting on $\mathcal{H}_{k i n}$ such that as $l \rightarrow 0$ we can express $\left\langle n\left|\hat{P}_{l, \epsilon}\right| m\right\rangle$ as a sum over triangulations with the correct weights. In other words we would like to deduce the appropriate quantisation of the evolution $U_{l, \epsilon}(x)$ from the path integral form of the theory. To do this we will identify the action of the operator $\hat{U}_{l, \epsilon}(x)$ with the stacking of triangles.

There are two ways to add triangles to a sequence of links $|n\rangle$. We can glue one or two faces of the triangle to the boundary. This creates a new set of links $|n+1\rangle$ or $|n-1\rangle$ respectively. Roughly, we will define $\hat{U}_{l, \epsilon}(x)$ to be the sum of both these local actions as shown in figure 1. Repeated action of $\hat{U}_{l, \epsilon}$ will then create a triangulation. An important aspect of this identification (which is unique to the dynamical triangulations framework) is that the parameter $a$ in our model can be interpreted as a proper time distance. Hence it is natural to identify $a$ with $\epsilon$, as we will do in the following.

These ideas can be expressed more precisely in the dual picture, which is also more likely to be useful in generalisations to higher dimensions and spin-foams. In general, the dual of a $(n+1)$-dimensional triangulation is an $(n+2)$-valent graph. The graph has a node in the centre of each simplex and one edge crossing each face as shown in figure 2. In one dimension the dual of a triangulation is just a sequence of nodes. Hence states that are dual to an auxiliary state $\left|v_{1}, \ldots, v_{n}\right\rangle$ will be denoted by $|\vec{x}\rangle:=\left|x_{1}, . ., x_{n}\right\rangle$ where $x_{i}:=\left(v_{i}+v_{i+1}\right) / 2$ now is the co-ordinate of the $i$ 'th node which corresponds to the centre of the $i$ 'th link.

The operator $\hat{U}_{l, a}(x)$, being local, acts on auxiliary states. Let us introduce creation and annihilation operators $\hat{a}^{\dagger}(x)$ and $\hat{a}(x)$ acting on nodes of auxiliary 


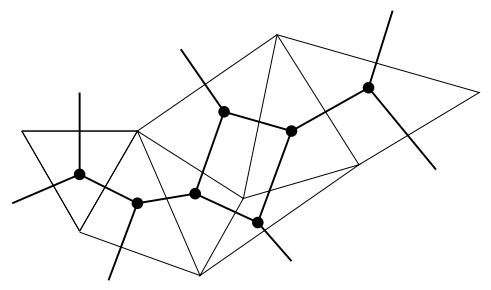

FIgURE 2. A 2-dimensional triangulation and its dual trivalent graph
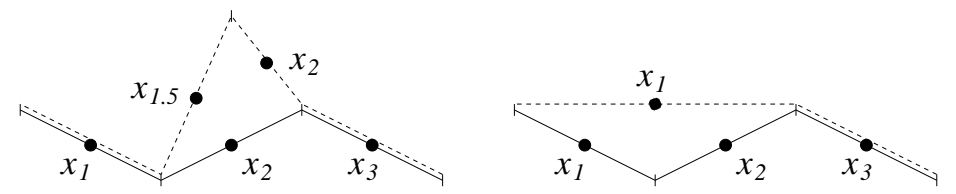

Figure 3. The actions of $\hat{a}^{\dagger}\left(x_{2}\right)$ (left fig.) and $\hat{a}\left(x_{2}\right)$ (right fig.) on the auxiliary state $\left|x_{1}, x_{2}, x_{3}\right\rangle$. The newly created states are depicted by the dashed lines and $x_{1.5}=\left(x_{1}+x_{2}\right) / 2$.

dual states:

$$
\begin{array}{r}
\hat{a}^{\dagger}(x)\left|x_{1}, \ldots x_{n}\right\rangle:= \begin{cases}0 & \text { if } x \neq x_{i} \\
\left|x_{1}, \ldots, x_{i-1}, \frac{x_{i-1}+x_{i}}{2}, x_{i}, \ldots, x_{n}\right\rangle & \text { if } x=x_{i}\end{cases} \\
\hat{a}(x)\left|x_{1}, \ldots, x_{n}\right\rangle:= \begin{cases}0 & \text { if } x \neq x_{i} \\
\left|x_{1}, \ldots, \not x_{i}, \ldots x_{n}\right\rangle & \text { if } x=x_{i}\end{cases}
\end{array}
$$

This can be identified with the gluing of triangles as follows. The creation operator $\hat{a}^{\dagger}\left(x_{i}\right)$ corresponds to the gluing of one edge of a triangle to a boundary link centred at $x_{i}$. This gives a new boundary triangulation, which is identified with an auxiliary state by assigning the the node corresponding to right free edge of the added triangle with the coordinate $x_{i}$ and the left edge with $\left(x_{i-1}+x_{i}\right) / 2$ as depicted on the left of figure 3 .

The annihilation operator $\hat{a}\left(x_{i}\right)$ acts by gluing two edges of a triangle to the boundary links $x_{i}$ and $x_{i-1}$. This gives a new state with one less link than the original one. The free edge of the added triangle is identified with the node $x_{i-1}$ as can be seen on the right of figure 3. We use these operators to define $\hat{U}_{l, a}(x)$ :

$$
\hat{U}_{l, a}(x)|\vec{x}\rangle:=\frac{1}{2} e^{-a^{2} \lambda}\left(\hat{a}^{\dagger}(x)+\hat{a}(x)\right)|\vec{x}\rangle
$$

Multiple actions of $\hat{U}_{l, a}(x)$ will thus give rise to a sum of auxiliary states, each of which can be identified with an abstract triangulation. The projector is given by a sum of ordered products $\hat{U}_{l, a}\left(x_{1}\right) \cdots \hat{U}_{l, a}\left(x_{s}\right)$ as shown in equation (19). In the $(1+1) \mathrm{d}$ case the ordering means that $x_{1} \leq x_{2} \leq \ldots \leq x_{s}$. This implies that an ordered product of evolution operators will construct triangles from right to left (if the coordinate axis points left to right).

The action of $\hat{U}_{l, a}(x)$ on a state $|\vec{x}\rangle$ will be 0 whenever $x$ is not the coordinate of a node in $|\vec{x}\rangle$. It follows that whenever the action of a product $\hat{U}_{l, a}\left(x_{1}\right) \cdots \hat{U}_{l, a}\left(x_{s}\right)$ is non-zero it will act by constructing a sum of triangulations, each containing $s$ 
triangles. Hence $\hat{P}_{l, a}$ corresponds to a sum over triangulations, where the parameter $s$ in equation (19) determines the number of triangles in a triangulation.

As $l \rightarrow 0$ the separation between the allowed values for $x \in S^{1}$ tends to 0 . Hence, in this limit, any triangulation that can be constructed by an ordered sequence of evolution operators will be present in the sum over triangulations described by $\hat{P}_{l, a}$

We now complete the definition of $\left\langle n\left|\hat{P}_{l, a}\right| m\right\rangle$ as follows. We choose an auxiliary state $|\vec{m}\rangle$ which is a representative in the class $|m\rangle$, i.e. any auxiliary state that has $m$ links. The action of $\hat{P}_{l, a}$ on this state will produce a sum of auxiliary states:

$$
\hat{P}_{l, a}|\vec{m}\rangle=\sum_{i} e^{-a^{2} \lambda N_{\mathbf{\Delta}_{i}}}\left|\vec{x}_{i}\right\rangle,
$$

where each of these states $\left|\vec{x}_{i}\right\rangle$ is the (future) boundary of an abstract triangulation $\boldsymbol{\Lambda}_{i}$ containing $N_{\mathbf{\Lambda}_{i}}$ triangles as described above. This gives:

$$
\left\langle n\left|\hat{P}_{l, a}\right| m\right\rangle=\sum_{i} e^{-a^{2} \lambda N_{\mathbf{\Delta}_{i}}}\left\langle n, \vec{x}_{i}\right\rangle
$$

The inner product $\left\langle n, \overrightarrow{x_{i}}\right\rangle$ is given by the dual action of $|n\rangle$ on the auxiliary state $\left|\overrightarrow{x_{i}}\right\rangle$. This is 1 if if the number of links in $\left|\vec{x}_{i}\right\rangle$ is $n$ and 0 otherwise.

In this way $\left\langle n\left|\hat{P}_{l, a}\right| m\right\rangle$ is given by a sum over triangulations that have two boundaries, with $n$ and $m$ links respectively. The amplitude for each triangulation is just $e^{-a^{2} \lambda N_{\mathbf{\Delta}}}$, where $N_{\mathbf{\Delta}}$ is the number of triangles in the triangulation as required.

Given two different auxiliary states representing the same kinematical state (i.e. having the same number of nodes), we can find two ordered sequences of operators that will produce the same abstract sum of triangulations when acting on the respective states. This is because the coordinates of the nodes just serve to label the links and their exact value is irrelevant for the geometrical interpretation of the action of $\hat{U}_{l, a}(x)$. But as noted above, in the limit that $l \rightarrow 0$, any triangulation that can be constructed by an ordered sequence of evolutions acting on a given auxiliary state, will be present in the expansion of $\hat{P}_{l, a}$. Hence, in this limit, the amplitude becomes independent of the choice of representative for the state $|m\rangle$. For the same reason the amplitude also becomes independent of the metric chosen in the regularisation of $\hat{P}_{l, a}$

To conclude we note that the triangulations constructed with the above definitions are unique, i.e. two different sequences of operators $\hat{U}_{l, a}\left(x_{1}\right) \cdots \hat{U}_{l, a}\left(x_{s}\right)$ acting on the same auxiliary state will give rise to different triangulations. This follows essentially from the ordering of the operators as shown in the appendix. However it is also clear that not all triangulations are produced c.f. figure 1 . This is a feature of the choice of definition of $\hat{U}_{l, a}(x)$. Indeed, it is possible to choose a different action of $\hat{U}_{l, a}(x)$ in which $\hat{a}\left(x_{i}\right)$ corresponds to a gluing of a triangle to the links $x_{i}$ and $x_{i+1}$. In this case all triangulations are produced albeit not uniquely. It remains to be seen if an action can be chosen such that we have both properties. In this respect it will be important to check to which universality class the continuum limit of the triangulations model constructed above corresponds to. As we discuss below the derivation of the Hamiltonian corresponding to this model suggests that we are summing over geometries that suppress the creation of baby universes. 


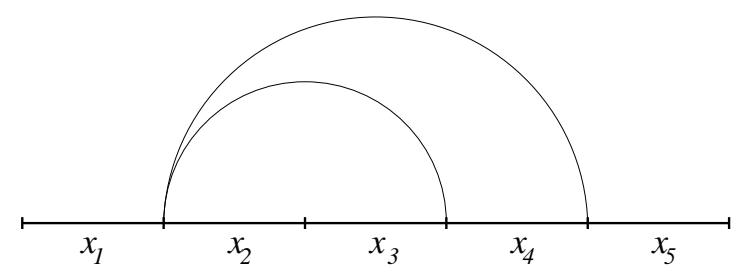

Figure 4. This triangulation cannot be constructed with an ordered sequence of $\hat{U}_{l, \epsilon}(x)$. This is because the top triangle has to be constructed after the bottom one. This cannot happen since it is situated to the right. In other words we would need a sequence $\hat{U}_{l, a}\left(x_{4}\right) \hat{U}_{l, a}\left(x_{3}\right)$, which is not allowed since $x_{4} \geq x_{3}$.

3.3. The Hamiltonian. One of the benefits of working in $1+1$ dimensions is that we can use the definition of $\hat{U}_{l, a}(x)$ to derive the form of the continuum, averaged, Hamiltonian constraint, thus completing the link between the covariant and canonical pictures .

The Hamiltonian and $\hat{U}_{l, a}(x)$ are related via: $\hat{U}_{l, a}(x)=e^{-a l \hat{H}(x)}$. In the following we will take the $a$ and $l$ limits simultaneously and hence set $a=l$. Thus for small $a$ we have: $\hat{U}_{a}(x) \approx 1-a^{2} \hat{H}(x)$. Let us define the averaged Hamiltonian as:

$$
\bar{H}_{a}=\int_{S^{1}} d x \hat{H}_{a}(x)
$$

Then

$$
a \hat{S}_{a}:=\int_{S^{1}} d x \hat{U}_{a}(x) \approx \hat{L}\left(S^{1}\right)-a^{2} \bar{H}_{a},
$$

where $L\left(S^{1}\right)$ denotes the length of the spatial slice $S^{1}$. The point is that the integrated evolution operator $\hat{S}_{a}$ has a simple expression. It is the sum of the elementary evolution actions (adding of a triangle in two possible ways) on every node of a state $|n\rangle$. More specifically, the matrix elements of $\hat{S}_{a}$ are given by

$$
\left\langle n\left|\hat{S}_{a}\right| m\right\rangle=\frac{m}{2} e^{-a^{2} \lambda}\left(\delta_{m, n-1}+\delta_{m, n+1}\right)
$$

The length $\hat{L}\left(S^{1}\right)$ also has a natural operator action. Since the states $|n\rangle$ describe geometries of length $n a$ it follows that $|n\rangle$ are volume eigenstates, i.e.:

$$
V(\Sigma)|n\rangle=n a|n\rangle .
$$

Hence from equations (26), (27) and (28) we learn that the matrix elements of the discretised averaged Hamiltonian operator should be given by:

$$
\left\langle n\left|\bar{H}_{a}\right| m\right\rangle \approx \frac{m}{a}\left(\delta_{m, n}-\frac{1}{2} e^{-a^{2} \lambda}\left(\delta_{m, n-1}+\delta_{m, n+1}\right)\right)
$$

The states $|n\rangle$ describe geometries of length $N=$ an. We want to take the limit $a \rightarrow 0$ while keeping $N$ fixed. In this way we obtain a continuum Hilbert space spanned by the states $|N\rangle$. We are looking for the continuum expression $\bar{H}$ of $\bar{H}_{a}$

${ }^{6}$ We thank Jan Ambjørn for this observation. 
which acts on this space i.e. we are interested in:

$$
\bar{H} f(N)=\int d M\langle N|\bar{H}| M\rangle f(M)
$$

Since the discrete states $|n\rangle=|N / a\rangle,|m\rangle=|M / a\rangle$ become increasingly better approximations to the continuum states $|N\rangle$ and $|M\rangle$ as $a \rightarrow 0$, we can replace the above by:

$$
\bar{H} f(N)=\lim _{a \rightarrow 0} \sum_{m}\left\langle n\left|\bar{H}_{a}\right| m\right\rangle f(m a)
$$

Using the fact that $n=N / a$ and $m=M / a$ together with equation (29) we obtain

$$
\bar{H} f(N)=\lim _{a \rightarrow 0} \frac{N}{a^{2}}\left(f(N)-\frac{1}{2} e^{-a^{2} \lambda}(f(N-a)+f(N+a))\right)
$$

Expanding $f(N-a), f(N+a)$ to second order in $a$ and also expanding the exponential this gives

$$
\begin{aligned}
\bar{H} f(N) & =\lim _{a \rightarrow 0} \frac{N}{a^{2}}\left(f(N)-\frac{1}{2}\left(1-a^{2} \lambda\right)\left(2 f(N)+a^{2} \frac{d^{2}}{d N^{2}} f(N)+\mathcal{O}\left(a^{3}\right)\right)\right) \\
& =N \lambda f(N)-N \frac{1}{2} \frac{d^{2}}{d N^{2}} f(N)
\end{aligned}
$$

Hence we deduce

$$
\bar{H}=\lambda N-N \frac{1}{2} \frac{d^{2}}{d N^{2}}
$$

This is the same expression (up to a factor of 2) that has been obtained in the study of dynamical triangulation models [1]. It corresponds to a choice of triangulation models in which baby universe creation is not allowed [?

3.4. Discussion. We have succeeded in deriving the form of the averaged Hamiltonian constraint operator from the regularised path integral for $1+1$ quantum gravity using our projector expansion. This hinged crucially on the identification of the elementary evolutions with the addition of triangles. Moreover, this allowed us to naturally identify the small proper time step $a$ occurring in the regularisation of the projector with the edge length of the triangles. This gives rise to an interpretation to the integral over proper times $[d T(x)]$ as a sum over triangulations.

These features are not present in the original regularisation of the projector proposed by Reisenberger and Rovelli [20]. They suggested an expansion of the exponential in the projector

$$
\hat{P}=\int[d T] \sum_{t=0}^{\infty} \frac{\left(-\hat{H}[T]^{t}\right)}{t !},
$$

which as we will discuss later this was motivated by the form of the Hamiltonian constraint in loop quantum gravity. In order for this expression to be interpretable as a sum over triangulations it turns out that we need to identify $\hat{H}[T]$ with the

7 Durhuus and Lee have shown [6] that $\lim _{a \rightarrow 0}\left\langle N / a\left|e^{-T \bar{H}_{a}}\right| M / a\right\rangle=G(N, M ; T)$. Here $G(M, N ; T)$ denotes a sum over sliced triangulations with $T$ slices as depicted in figure 10 (see 11 for details). From our perspective it is clear why this should be so. We have $e^{-T \vec{H}}=\exp \left[-T \int_{S^{1}} d x \hat{H}_{a}(x)\right]=\left[\prod_{x \in S^{1}} \hat{U}_{a}(x)\right]^{T}$. Hence the action of this operator corresponds to the stacking of $T$ layers of triangles to a boundary triangulation. 
integrated move operator $\hat{S}_{a}$. In this way multiple action of the Hamiltonian will produce all possible triangulations.

This leaves the interpretation of the $[d T(x)]$ integral unclear since a sum over all triangulations is already generated for every function $T(x)$. Intuitively, the proper time should be reflected in the triangulation. Indeed in the dynamical triangulations approach it is clear that the proper time corresponds to the number of edges of triangles along a particular path.

Additionally, a problem arises because there are many ways in which a given triangulation can be constructed by a sequence of stacking of triangles. All these possibilities will be included in the sum generated by the above expansion ${ }^{8}$. This number is a non-trivial function of the triangulation and does not depend solely on $t$, the number of triangles in the triangulation. Hence the sum over triangulations will contain non-trivial symmetry factors that do not occur in the definition of $G_{a}\left(\triangle, \Delta^{\prime}\right)$.

Crucially, however, because one has made the identification $H[T]=\hat{S}_{a}$ it will no longer be possible to derive the correct expression for the continuum Hamiltonian.

3.5. Generalisations. It is straightforward to generalise our discussion to higher dimensions. Space-time triangles will be replaced by $(n+1)$-simplices, where $(n+1)$ is the space-time dimension. These can be glued in $(f-1)=(n+1)$ ways to a given boundary triangulation, where $f$ is the number of faces of the simplex. These moves will be identified with the action of the local evolution operator and in this way we can again interpret the projector as a sum over triangulations. This is described in more detail for $(2+1) \mathrm{d}$ in the next section.

However in higher dimensions boundary states no longer only depend on the number of boundary simplices but also their connectivity, which is related in a complicated way to the limiting continuum geometry of a discrete state. This makes it very difficult to derive the action of a Hamiltonian differential operator. These issues are currently under investigation

\section{SPIN-FOAM MODELS AND LOOP QUANTUM GRAVITY}

We will now discuss spin-foam models of $B F$ theory and quantum gravity. In general, a spin-foam is a 2-dimensional complex with faces labelled by representations and edges labelled by intertwining operators. These structures were first used in path-integrals for quantum gravity by Reisenberger [19] and now provide a unifying framework for many approaches to quantum gravity (see [17] and [3] for reviews and references).

In order to make contact with our discussion of dynamical triangulations, we will make use of the fact that the dual of a spin-foam is a triangulation of space-time with labelled edges. We will see how one can provide a definition of the projector (not sum over geometries) in terms of a sum over such space-time triangulations. In contrast to the dynamical triangulations framework, however, we also sum over the labellings of the edges in this approach. We will show how a new regularisation of the projector will lead naturally to this class of models. The focus will be on the $(2+1) \mathrm{d}$ case, and we indicate at the end how the results can be generalised.

In $2+1$ dimensions general relativity is equivalent to a topological $B F$ theory due to the absence of local degrees of freedom or gravitons. In the Euclidean case

${ }^{8}$ This is avoided in our approach because the triangles are stacked in ordered sequences. 
the fundamental variables are an su(2) Lie algebra-valued space-time connection $\mathbf{A}$ and an su(2)-valued one form $\mathbf{B}$. The action is given by:

$$
S[\mathbf{B}, \mathbf{A}]=\int_{\mathcal{M}} \operatorname{Tr}[\mathbf{B} \wedge F(\mathbf{A})]
$$

where $F(\mathbf{A})$ is the curvature of $\mathbf{A}$. By restricting to space-time manifolds of the form $\mathcal{M}=\Sigma \times \mathbb{R}$, where $\Sigma$ is a compact 2-dimensional spatial manifold, we can pass to a Hamiltonian formulation. The configuration space variable is given by the restriction $A$ of the connection $\mathbf{A}$ to $\Sigma$. The Hamiltonian is a sum of two constraints? The first imposes $S U(2)$-gauge invariance and the second, also referred to as the curvature constraint, implies that $F(A)=0$, i.e. that the connection is flat. A good overview and a comprehensive list of references can be found in [5].

It can be shown that the above is equivalent to a Chern-Simons gauge theory which was first used by Witten in [34] to obtain a quantisation of $2+1$ gravity. Here we will be using the discrete Ponzano-Regge [18] or Tuarev-Viro [33] approaches to quantisation as they will resemble the dynamical triangulations models discussed above.

4.1. Quantum theory. The kinematical framework for the Ponzano-Regge model can be described by loop quantum gravity as first noticed by Rovelli [22]. The kinematical Hilbert space $\mathcal{H}_{k i n}$ is constructed from a space of gauge-invariant wavefunctions on the configuration space of connections. This space is spanned by the so-called spin-networks [24], which are the natural generalisations of the Wilson loop.

Spin-networks are functions of connections with support on graphs embedded in space, which in $2+1$ we choose to be trivalent. More precisely, given any closed, oriented, trivalent graph $\Gamma$ with $n$ edges embedded in $\Sigma$ and a labelling of the edges by representations of $\mathrm{SU}(2)$ denoted by $\vec{\jmath}=\left\{j_{1}, \ldots, j_{n}\right\}$, we can construct a spin-network function $10 \Psi_{\Gamma, \vec{\jmath}}(A)$ as follows. Let us denote the holonomy of the connection $A$ along the edge $e \in \Gamma$ by $U_{e}[A]$. This can be thought of as an $\mathrm{SU}(2)$ group element. Thus for every edge $e$ labelled by a spin $j_{e}$ we can construct a matrix $\rho_{j_{e}}\left(U_{e}[A]\right)_{b}^{a}$, where $\rho_{j_{e}}$ is the spin- $j_{e}$ representation of $\mathrm{SU}(2)$. We can associate the index $a$ with the vertex to which the edge $e$ is directed and the index $b$ to the vertex at the other end of $e$. Taking the tensor product of these matrices gives us a tensor with three indices for every vertex in $\Gamma$. We can contract these indices with the unique invariant tensor in the tensor product of the representations on the edges incident on the vertex. This gives us the gauge-invariant function $\Psi_{\Gamma, \vec{\jmath}}(A)$.

An inner product can now be imposed on the space generated by these functions by demanding that spin-networks be orthonormal, i.e.:

$$
\left\langle\Psi_{\Gamma, \vec{\jmath}}, \Psi_{\Gamma^{\prime}, \vec{\jmath}^{\prime}}^{\prime}\right\rangle_{k i n}=\delta_{\Gamma, \Gamma^{\prime}} \delta_{\vec{\jmath}, \vec{\jmath}^{\prime}}
$$

Completion with respect to this inner product gives us the kinematical Hilbert space $\mathcal{H}_{\text {kin }}$.

\footnotetext{
${ }^{9}$ Note that there is not need for a separate 2-diffeomorphism constraint as such transformations are already generated by the curvature constraint.

${ }^{10}$ Note that because we have chosen graphs to be trivalent we do not require a choice of intertwiner for the vertices. In $2+1$ this does not imply a loss of generality since the theory can be solved exactly in terms of these spin-networks. Whether this is also possible in $3+1$ dimensions with 4 valent graphs is unclear.
} 
We can also describe the trivalent spin-networks in terms of their dual triangulations $\Delta$, making the link to earlier discussions. Each edge of $\Delta$ is labelled by the spin on the edge in $\Gamma$ intersecting it. We will switch between these two perspectives whenever convenient and spin-networks will be labelled either by graphs, triangulations or not at all.

To proceed we now need to implement the Hamiltonian or curvature constraint $\hat{H}$ and reduce to the final, physical, Hilbert space $\mathcal{H}_{\text {phys }}$. As before this is done by constructing the generalised projector. In contrast to the previous section one can give an exact expression of this projector in terms of sums over triangulations.

As shown by Ooguri [16], the Ponzano-Regge model defines the projector for $2+1$ quantum gravity. This is most easily introduced in terms of triangulations $\boldsymbol{\Delta}$ of $\mathcal{M}=\Sigma \times[0,1]$. We will distinguish between the interior edges $\mathbf{e}$ and the edges $e$ of the boundaries $\Delta_{0}$ and $\Delta_{1}$ of $\boldsymbol{\Lambda}$. All edges are labelled by representations $j_{e}$ of $\mathrm{SU}(2)$. Hence we can associate to every tetrahedron $T \subset \boldsymbol{\Delta}$ a normalised 6-J symbol denoted by $T\left\{\vec{\jmath}_{T}\right\}$, where $\vec{\jmath}_{T}$ is the set of spins on the boundary edges of $T$, which can include edges that lie on the boundary of $\boldsymbol{\Lambda}$. To every triangulation $\Delta$ with given boundary $\Delta_{0}, \Delta_{1}$ we can now define an amplitude $Z_{\mathbf{\Lambda}}\left(\Delta_{0}, \Delta_{1}\right)$ as a sum over all possible labellings of the interior edges that are compatible with the labelling of the boundary:

$$
Z_{\mathbf{\Delta}}\left(\triangle_{0}, \Delta_{1}\right):=\prod_{e} \sqrt{2 j_{e}+1} \sum_{j_{\mathbf{e}}} \prod_{\mathbf{e}}\left(2 j_{\mathbf{e}}+1\right) \prod_{T} T\left\{\vec{\jmath}_{T}\right\} .
$$

This sum can be infinite and needs to be regulated. This can be done by introducing a cut-off as is done by Ooguri in [16 by using quantum groups. The latter approach gives the Tuarev-Viro model [33] which is related to quantum gravity with a cosmological constant. This will not concern us in the following and we assume that some choice of regularisation has been made to make the above expression well-defined.

Given two spin-networks $\Psi_{0}$ and $\Psi_{1}$, dual to triangulations $\Delta_{0}$ and $\Delta_{1}$, we can now define the physical inner product as a sum over triangulations $\boldsymbol{\Delta}$ that have boundary $\Delta_{0}+\Delta_{1}$ :

$$
\left\langle\Psi_{1}, \hat{P} \Psi_{0}\right\rangle_{k i n}=\sum_{\mathbf{\Delta}} Z_{\mathbf{\Delta}}\left(\triangle_{0}, \Delta_{1}\right)
$$

Using identities between the 6-J symbols one can show that $Z_{\mathbf{A}}\left(\Delta_{0}, \Delta_{1}\right)$ is in fact independent of the triangulation $\boldsymbol{\Delta}$ and depends only on $\Delta_{0}$ and $\Delta_{1}$. Hence the matrix elements of $\hat{P}$ are given by $\infty \times Z\left(\Delta_{0}, \Delta_{1}\right)$, where $Z$ is evaluated using any chosen triangulation with the correct boundary data. Such an infinite factor is a common feature in the RAQ procedure whenever the constraints do not form the Lie-algebra of a compact group 19 . The factor can be dropped by redefining the physical inner product and we have the final result 12 :

$$
\left\langle\tilde{\Psi}_{1}, \tilde{\Psi}_{0}\right\rangle_{\text {phys }}=Z\left(\triangle_{0}, \Delta_{1}\right) .
$$

\footnotetext{
${ }^{11}$ Recall that the projector averages over the action generated by the Hamiltonian constraint.

${ }^{12}$ The reason that we chose to include a sum over triangulations initially is that it will play an important role in the following. The above procedure is also necessary for gravity in higher dimensions where we no longer have triangulation independence.
} 
A crucial property for the identification of the amplitude $Z$ with a projector is the property:

$$
\sum_{j} Z\left(\triangle_{1}, \triangle_{j}\right) Z\left(\triangle_{j}, \Delta_{0}\right)=Z\left(\triangle_{1}, \Delta_{0}\right)
$$

where the sum is over all colourings $\Delta_{j}$ of an intermediate triangulation $\triangle$.

As has been shown by Ooguri [16], this definition of $\hat{P}$ leads to a well-defined theory of quantum gravity, which is equivalent to Witten's Chern-Simons approach. If we formulate the above in terms of spin-networks instead of their dual triangulations we obtain the spin-foam model of $2+1$ loop quantum gravity. A spin-foam is simply the 2-skeleton of the dual 2-complex of a given triangulation.

The sum over spins on a triangulation can be given an interpretation as a sum over geometries or path integral in several ways. Originally the Ponzano-Regge model was an ad hoc modification of the Regge model of gravity. Here one describes (piecewise linear) metrics by an assignment of edge lengths and angles to the tetrahedra in a triangulation of space-time. By restricting edge lengths to take on only integer and half-integer values we obtain the Ponzano-Regge model.

The actions of the two models can be related in a semi-classical limit where we take spins $j$ to be large. In this limit the amplitude for a single tetrahedron given by the $6-\mathrm{J}$ symbol can expressed as:

$$
T\left\{\vec{\jmath}_{T}\right\} \sim\left(6 \pi V_{T}\right)^{-1 / 2}\left(e^{-i\left(S_{R}+\pi / 4\right)}+e^{i\left(S_{R}+\pi / 4\right)}\right),
$$

where $V_{T}$ is the volume of the tetrahedron sides of length $L=\frac{1}{2}\left(j+\frac{1}{2}\right)$ and $S_{R}$ is the Regge action. Hence it is natural to identify spins with geodesic lengths in the Ponzano-Regge model. The fact that the 6-J symbol describes a sum of two exponentials with opposite signs is related to the fact that the Ponzano-Regge model defines a true projector and hence not a sum over geometries as we comment on again below.

A perhaps better understanding of the origin of the discreteness in the edge lengths comes via the spin foam approach. It is possible to show that PonzanoRegge model arises if one discretises the path integral:

$$
\int[d \mathbf{B}][d \mathbf{A}] e^{-i S[\mathbf{B}, \mathbf{A}]},
$$

where the action is defined in equation (37). The spins labelling edges then arise after an integration over $\mathbf{B}$, as the Fourier components of the connection $\mathbf{A}$ restricted to the edges of the triangulation. Furthermore, one can construct a length operator for loop quantum gravity and one finds that the spins are precisely proportional to the quantised edge lengths as first noticed by Rovelli in 22.

Since spin-foam models involve a sum over edge lengths, a continuum limit cannot be taken in the same way as in the dynamical triangulations models where edge lengths are taken to 0 . The implications of this will be discussed below. It turns out that we need to take the scale of the edge lengths to 0 . Note however that because the special cases of $B F$ theory and $2+1$ quantum gravity are topological these models can be defined exactly in terms of a single triangulation without taking a continuum limit as described above.

Note also that the sum over geometries that is described by the Ponzano-Regge and more general spin-foam models is not gauge fixed. In other words different spin assignments of a triangulation can correspond to the same geometry. The rationale 


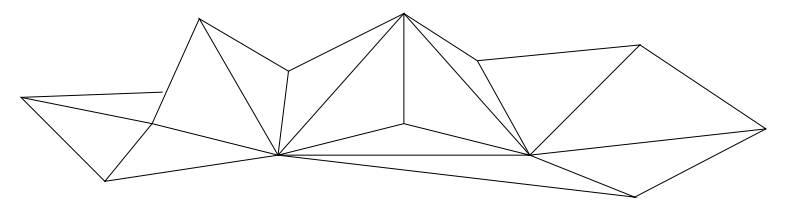

FiguRe 5. A boundary triangulation $\Delta_{0}$.

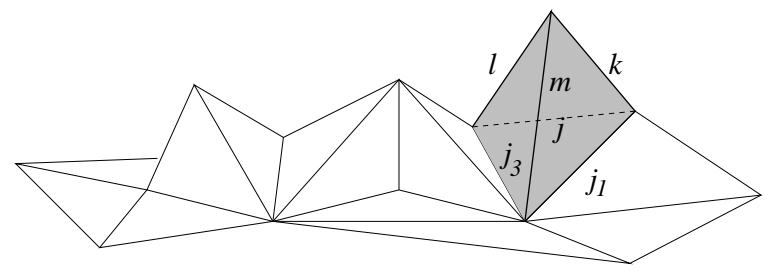

Figure 6 . Gluing one face a tetrahedron to $\Delta_{0}$. Only the relevant spins are labelled.

behind the spin foam approach is that instead of a picking a representative of each gauge equivalence class and integrating only over these we are in effect averaging over the equivalence classes. This will be important later.

4.2. The projector and elementary moves. In order to derive the sum over triangulations picture from the formal definition of the projector we now describe how one can construct $Z_{\mathbf{\Lambda}}\left(\Delta_{0}, \Delta_{1}\right)$ for any $\boldsymbol{\Lambda}$ and $\Delta_{1}$ given an initial triangulation $\triangle_{0}$ by an iterative sequence of elementary moves. These are the Pachner moves in $2+1$ dimensions by which all triangulations can be related.

There are 3 ways of gluing a tetrahedron $T$ onto a given triangulation $\Delta_{0}$ of $\Sigma$. These are shown in figures 6 , 7 and 8 :

1. We can attach one face of $T$ to a triangle in $\Delta_{0}$. To this tetrahedron we associate the factor

$$
T\left\{\begin{array}{ccc}
j_{1} & j_{2} & j_{3} \\
l & m & k
\end{array}\right\} \sqrt{(2 k+1)(2 l+1)(2 m+1)}
$$

where we have written out the 6 -J symbol and $k, l, m$ are the spins on the edges of $T$ that are not glued to $\triangle_{0}$

2. We can attach two faces of $T$ to $\Delta_{0}$. This is associated with a factor

$$
T\left\{\begin{array}{lll}
j_{1} & j_{3} & l \\
j_{4} & j_{2} & k
\end{array}\right\} \sqrt{(2 k+1)(2 l+1)}
$$

where $k$ labels the edge that lies in the interior of the two triangles glued to $\triangle_{0}$ and $l$ is the spin on the opposite edge.

3. We can glue three faces of $T$ to $\Delta_{0}$. In this case we have a factor of

$$
T\left\{\begin{array}{ccc}
j_{1} & j_{2} & j_{3} \\
l & m & k
\end{array}\right\} \sqrt{(2 k+1)(2 l+1)(2 m+1)}
$$

where $k, l, m$ label the edges in the interior of the three triangles attached to $\Delta_{0}$. 


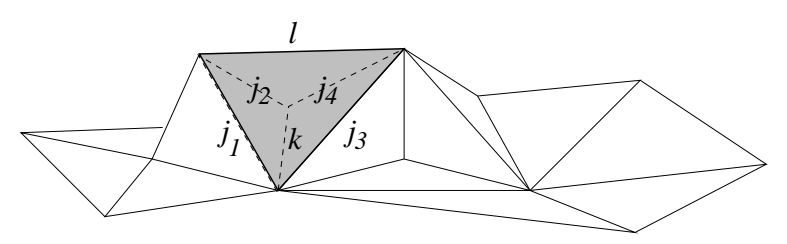

FIgURE 7. Gluing two faces of a tetrahedron to $\Delta_{0}$.

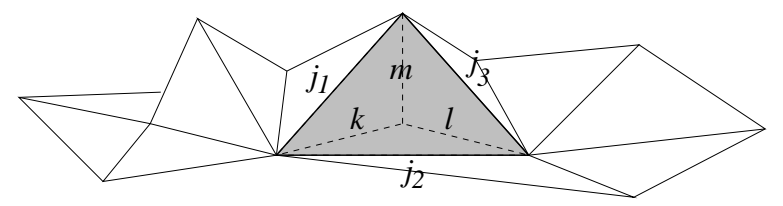

Figure 8 . Gluing 3 faces of a tetrahedron to $\Delta_{0}$.

Once we have completed the gluing ${ }^{13}$ we obtain a new boundary triangulation $\triangle^{\prime}$. Using this boundary we can repeat the above procedure. In this way we can construct any triangulation $\boldsymbol{\Delta}$ with any boundary $\Delta_{1}$ given an original boundary $\triangle_{0}$. By taking the product of the factors associated to the tetrahedra we recover the amplitude $Z_{\mathbf{\Delta}}\left(\triangle_{0}, \Delta_{1}\right)$.

Equivalently such a procedure can be viewed as constructing a sequence of spinnetworks via a sequence of operator actions, which can be formalised as follows. We introduce the 3 move operators $\hat{m}_{1}, \hat{m}_{2}$ and $\hat{m}_{3}$, that act locally on spin-networks:

$$
\begin{aligned}
& \hat{m}_{1} \sum_{j_{2}}^{j_{1}}=\sum_{k, l, m} T\left\{\begin{array}{ccc}
j_{1} & j_{2} & j_{3} \\
l & m & k
\end{array}\right\} \sqrt{(2 k+1)(2 l+1)(2 m+1)}
\end{aligned}
$$

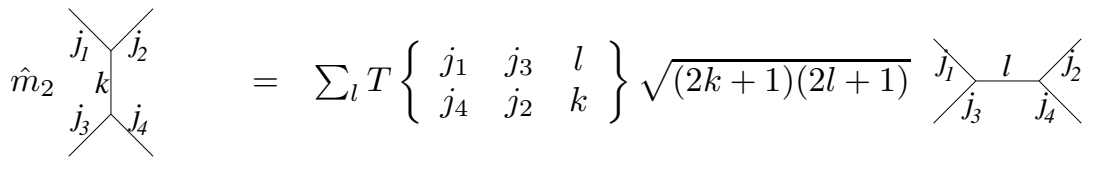

$$
\begin{aligned}
& \hat{m}_{3} \quad k \widehat{k}_{j_{2}}^{j_{l}} m=\left.T\left\{\begin{array}{ccc}
j_{1} & j_{2} & j_{3} \\
l & m & k
\end{array}\right\} \sqrt{(2 k+1)(2 l+1)(2 m+1)} j_{2}\right|_{j_{3}} ^{j_{1}}
\end{aligned}
$$

By a finite sequence of such moves one can relate any two trivalent spin-networks. Note that $\hat{m}_{1}, \hat{m}_{2}$ and $\hat{m}_{3}$ are precisely dual to the three possible gluings of tetrahedra as shown in figure 9. The amplitudes also correspond to those given in equations (45), (46) and (47).

\footnotetext{
${ }^{13}$ In terms of spin-foams we are gluing together elementary atoms as is described in 21]. The different ways of gluing triangles correspond to the choice of which boundary edges of the atom we wish to glue to the given spin-network.
} 


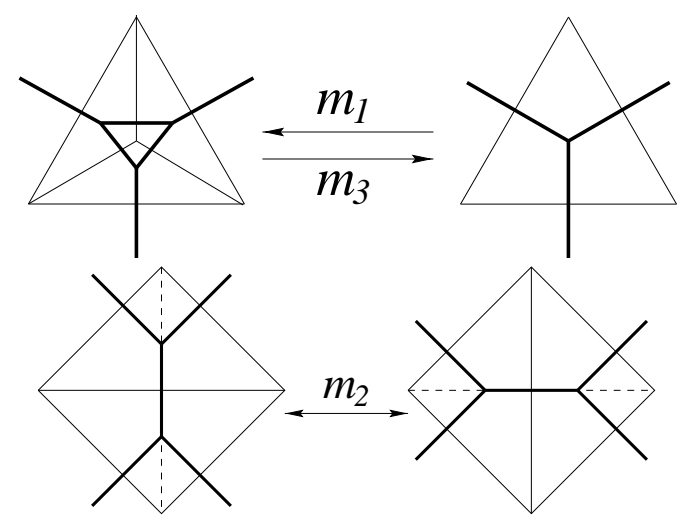

FiguRE 9. The action of the elementary moves on spin networks are dual to the addition of tetrahedra. Tetrahedra are depicted by the thin lines and are viewed from the top. For example, we can view top left figure as boundary triangulation consisting of 3 triangles with its dual graph. By gluing a tetrahedron to all three triangles we obtain a new boundary triangulation consisting of just one triangle as shown on the right. This corresponds to the move $\hat{m}_{3}$ acting on the dual graphs.

Hence any coloured triangulation $\boldsymbol{\Delta}$ can be viewed as a given sequence of such elementary moves.

As with the dynamical triangulations models, we wish to use the fact that the projector can be expressed as an ordered sequence of operations in order to derive the above action from the formal expression of the projector. This will clarify how the elementary move operators $\hat{m}_{1}, \hat{m}_{2}, \hat{m}_{3}$ are related to evolution operators and in consequence the Hamiltonian.

4.3. Regularisation of the projector II. We wish to provide a regularisation of the generalised projector as a product of elementary local operators in analogy to the approach in section 3.1. These elementary operators are to be identified with the moves $\hat{m}_{1}, \hat{m}_{2}$ and $\hat{m}_{3}$. But note that in contrast to the dynamical triangulations case the these moves do not involve a fixed proper time, instead the describe a sum over all possible proper times. This needs to be reflected in our expansion.

It turns out that the correct starting point for our calculation is the following expression for the projector:

$$
\hat{P}:=\int[d N(x, t)] e^{-i \int_{0}^{1} d t \int d^{2} x N(x, t) \hat{H}(x)},
$$

where we are integrating over all lapse functions on the cylinder $\Sigma \times[0,1]$. The reason we need to extend the range of integration as compared to our previous definition in equation (3) is that the path integral that we wish to recover is not gauge fixed as mentioned already above. This is reflected in the fact that the two definitions of the projector are related by:

$$
\int[d N(x, t)] e^{-i \int_{0}^{1} d t \int d^{2} x N(x, t) \hat{H}(x)}=\mathcal{N} \int[d T(x)] e^{-i \hat{H}[T]},
$$


where $T(x)=\int_{0}^{1} d t N(x, t)$ and $\mathcal{N}$ is an infinite normalisation factor, which should correspond to the volume of the gauge equivalence classes. Finally, we note that in this section we integrate over the entire range of lapse functions since we want to describe a genuine projector.

Let us now proceed with the regularisation of $\hat{P}$ by replacing integrals by Riemann sums. First we consider the integral over the coordinate time $t$ and we replace the integration over functions $N(x, t)$ by a product of integrations over spatial functions $N(x)$. This leads to the following regularised expression:

$$
\begin{aligned}
\hat{P}_{\epsilon} & =\int[d N(x, t)] e^{-i \sum_{t=0}^{k} \epsilon \int d^{2} x N(x, t \epsilon) \hat{H}(x)} \\
& =\prod_{t=0}^{k} \int[d N(x)] e^{-i \epsilon \int d^{2} x N(x) \hat{H}(x)}
\end{aligned}
$$

where $k=1 / \epsilon$. Now we proceed analogously with the remaining spatial integral in the exponential. To do this we need to introduce a square lattice with a set of vertices $V$ as before.

$$
\begin{aligned}
\hat{P}_{l, \epsilon} & =\prod_{t=0}^{k} \int[d N(x)] e^{-i \epsilon \sum_{x \in V} l^{2} N(x) \hat{H}(x)} \\
& =\prod_{t=0}^{k} \prod_{x \in V} \int_{-\infty}^{\infty} d T e^{-i \epsilon l^{2} T \hat{H}(x)} \\
& =\prod_{t=0}^{k} \prod_{x \in V} \int_{0}^{\infty} d T\left(e^{-i \epsilon l^{2} T \hat{H}(x)}+e^{i \epsilon l^{2} T \hat{H}(x)}\right),
\end{aligned}
$$

where we are left with a regular integral over $T \in \mathbb{R}$. Let us now set

$$
\hat{U}_{l, \epsilon}(x):=\int_{0}^{\infty} d T\left(e^{-i \epsilon l^{2} T \hat{H}(x)}+e^{i \epsilon l^{2} T \hat{H}(x)}\right)
$$

then we have the final expression:

$$
\hat{P}_{l, \epsilon}=\prod_{t=0}^{k} \prod_{x \in V} \hat{U}_{l, \epsilon}(x)
$$

This expression is similar to equation (19) and can be interpreted as a sum over triangulations. There are however essential differences, most notably in the definition of $\hat{U}_{l, \epsilon}$. Let us take these in turn.

4.4. Interpretation. The operator $\hat{U}_{l, \epsilon}(x)$ we have defined above is given by a sum over local evolution operators for all proper times. Hence it is natural to make the identification:

$$
\hat{U}_{l, \epsilon}(x)=\hat{m}_{1}+\hat{m}_{2}+\hat{m}_{3}
$$

The argument $x$ of the operator $\hat{U}_{l, \epsilon}(x)$ determines the location of the action of the move operators $\hat{m}_{i}$ on a given spin network. The integral over $T$ in the definition of $\hat{U}_{l, \epsilon}$ essentially reflects the sum over spins in the definition of the elementary moves. This identification is further supported by the fact that $\hat{U}_{l, \epsilon}$ contains two terms involving an evolution in opposite proper time directions. This mirrors the semi-classical interpretation of the 6-J symbol given a sum of two exponentials of the Regge action described in equation (43). Here we can see that this is a result 


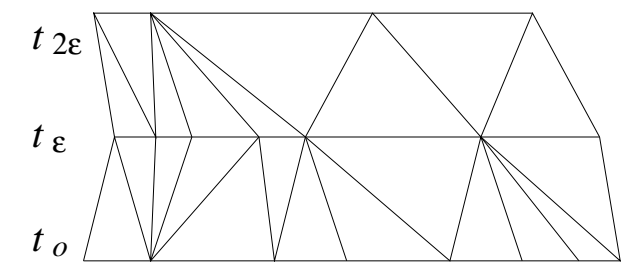

Figure 10. A $(1+1)$ d triangulation with a slicing structure.

of the integration range in the integral over lapse functions in the definition of the projector and hence a consequence of the fact that we are not dealing with a sum over geometries but a genuine projection operator.

Once we have made the identification of $\hat{U}_{l, \epsilon}$ with the elementary moves we can see how equation (56) describes a sum over triangulations. Each operator $\hat{U}_{l, \epsilon}(x)$ adds a tetrahedron in all possible ways and with all possible spins to a boundary triangulation at location $x$. As we take the limit $l \rightarrow 0$ the product over positions $x \in V$ then describes the addition of tetrahedra to every position on the initial boundary, such that we obtain a single layer of tetrahedra. We then repeat this at the next co-ordinate time step. The result is that we obtain a sum over triangulations which each have a layered structure as depicted in figure 10.

These layers can be interpreted as the slices $\{t\} \times \Sigma$ in the foliation $\Sigma \times[0,1]$. The spins on the edges of the tetrahedra describe the multi-fingered proper-time separation between the slices. The sum over spins then includes all possible propertime separations between initial and final boundaries.

We have so far not addressed how the parameter $\epsilon$ is related to the moves $\hat{m}_{i}$ and how this continuum limit $\epsilon \rightarrow 0$ is to be interpreted. Indeed, as mentioned above we can no longer identify $\epsilon$ simply with a proper time separation since we are summing over all possible proper times for every tetrahedron. Instead we see from equation (55) that $\epsilon$ multiplies the proper time $T$ and hence is responsible for determining the scale of the sum over proper times. This indicates that $\epsilon$ should be identified with the scale associated in the relation between spins and edge lengths of tetrahedra. Indeed, in a continuum limit we would like the edge lengths to become small in some units. Hence, we should set physical lengths proportional to spins via: $L \sim \epsilon j$.

Remarkably this parameter arises naturally in $3+1$ loop quantum gravity. It is the Immirzi parameter [11, 25] $\gamma$, which describes a one-parameter family of classical canonical transformations which in turn give rise to a one-parameter family of inequivalent quantum theories. This parameter relates spin labels of spin networks to the spectra of physical geometrical operators. It determines that the eigenvalues are given in units of $\left(\gamma l_{P}\right)^{a}$ where $l_{p}$ is the Planck length and the power $a$ is the dimension of the geometric observable we are considering. Hence, we deduce that we should make the identification $\gamma=\epsilon$ and a continuum limit of loop quantum gravity is obtained by taking the Immirzi parameter to 0 . This is especially interesting as it has been proposed in an entirely different context by Bojowald 佨, who studied reduced cosmological models using loop quantum gravity.

We note again that $B F$ theory and $(2+1)$ d gravity are special in that they are topological and the limit $\epsilon \rightarrow 0$ does not in fact need to be taken to define the theory. This can be interpreted as stating that it does not matter at which scale we 
approximate a continuum geometry. Since there are only a finite number of degrees of freedom any discrete approximation will capture the full physical content of the theory.

4.5. The Hamiltonian. In the previous section we have provided a well motivated expansion of the projector describing the Ponzano-Regge sum over triangulations. Some of the attractive features of this approach were:

- The natural identification of the sum over spins as a sum over proper-times;

- The explanation of the semi-classical limit of 6-J symbols in equation (43) as a result of the integration range in the generalised projector;

- The identification of the continuum limit as a limit in which the scales of edge lengths are taken to 0 ;

- The reflection of the fact that the Ponzano-Regge model does not describe a gauge fixed sum over metrics in the definition of the projector given in equation (48).

In this section we discuss a further motivation for our approach, which is closely linked to questions concerning the Hamiltonian. The point is that the local move operators $\hat{m}_{i}$ leave physical states invariant, i.e.:

$$
\left\langle\tilde{\Psi}, \hat{m}_{1} \tilde{\Phi}\right\rangle_{p h y s}=\left\langle\tilde{\Psi}, \hat{m}_{2} \tilde{\Phi}\right\rangle_{p h y s}=\left\langle\tilde{\Psi}, \hat{m}_{3} \tilde{\Phi}\right\rangle_{p h y s}=\langle\tilde{\Psi}, \tilde{\Phi}\rangle_{p h y s}
$$

for all $\tilde{\Psi}, \tilde{\Phi}$ This is shown by Ooguri in 16. Let us take $\hat{m}_{2}$ as an example. We have:

$$
\hat{m}_{2} \Phi_{\triangle_{0}}=\sum_{k} T\left\{\vec{\jmath}_{T}\right\} \sqrt{(2 k+1)(2 j+1)} \Phi_{\Delta_{k}}=\sum_{k} Z\left(\triangle_{k}, \triangle_{0}\right) \Phi_{\Delta_{k}},
$$

Here $\Phi_{\Delta_{0}}$ is the spin-network dual to the coloured triangulation $\Delta_{0}$ and $\Delta_{k}$ is the triangulation which is obtained from $\Delta_{0}$ by adding a tetrahedron with external spin $k$ as shown in figure 6 . The last equality in the above equation follows from the definition of $Z$ and the fact that, due to the triangulation invariance of the model, the transition amplitude between the states $\Phi_{\Delta_{0}}$ and $\Phi_{\Delta_{l}}$ is given by the amplitude of a single tetrahedron, which in turn is the same as the amplitude of the $\hat{m}_{2}$ move.

Hence, it follows:

$$
\begin{aligned}
\left\langle\tilde{\Psi}_{\triangle_{1}}, \hat{m}_{2} \tilde{\Phi}_{\Delta_{0}}\right\rangle_{p h y s} & =\sum_{k} Z\left(\Delta_{1}, \Delta_{k}\right) Z\left(\Delta_{k}, \Delta_{0}\right) \\
& =Z\left(\Delta_{1}, \Delta_{0}\right)=\langle\tilde{\Psi}, \tilde{\Phi}\rangle_{p h y s},
\end{aligned}
$$

where we have used the projection property (42) of the amplitude $Z$.

This can also be understood as follows. The constraints of $2+1$ general relativity impose the condition that the connection is flat. Physical states can be though of spin-network functions evaluated on flat connections. But it can be shown that the action of the elementary moves on spin-network functions restricted to flat connections is the identity.

Physical states are orbits of kinematical states under the exponentiated action of the Hamiltonian constraint. Hence the elementary moves map between states in $\mathcal{H}_{\text {kin }}$ that are related by such action and they can thus be viewed as elementary time-evolutions. Thus it is natural to identify the moves with the evolution operator $\hat{U}_{l, \epsilon}$.

Note, however, that this is in contrast to earlier assumptions on the action of the Hamiltonian in loop quantum gravity. Let us recall that in the Reisenberger-Rovelli 
approach the exponential in the projector is expanded as follows:

$$
\int[d T(x)] e^{-i \hat{H}[T]}=\int[d T(x)] \sum_{t=0}^{\infty} \frac{(-i)^{t}}{t !} \hat{H}[T]^{t},
$$

The idea was to identify $\hat{H}[T]$ with the total move operator

$$
\hat{H}[T]=\hat{S}:=\int_{\Sigma} d x\left(a_{1} \hat{m}_{1}+a_{2} \hat{m}_{2}+a_{3} \hat{m}_{3}\right),
$$

where $a_{1}, a_{2}$ and $a_{3}$ are arbitrary coefficients that need to be fixed. This was motivated because the action of Thiemann's Hamiltonian 32 in $3+1$ dimensions resembled the move $\hat{m}_{1}$ albeit with a different amplitude. The other moves need to be included in the Hamiltonian if the multiple actions of the Hamiltonian are to give rise to all triangulations. This is because the integral over the scalar lapse functions does not alter the topological action of the operators. The search for such a Hamiltonian, also referred to as crossing symmetric, has met only with partial success [7]. In particular it is not clear how the move $\hat{m}_{2}$, which is thought to be crucial for long range propagation of excitations [26], can be derived from the quantisation of a classical expression of a Hamiltonian.

But from the discussion above one can understand why this might be the wrong approach. Since the local moves leave physical states invariant it is impossible that any combination of them will annihilate physical states as would be required if they were to correspond to a Hamiltonian constraint. This is because the integrated move operator will be a sum of elementary operators acting at all possible locations in the triangulation underlying a spin-network. Each single action leaves physical states invariant. This implies the following for the action of the Hamiltonian defined in equation (63) on spin networks $\Psi$ and $\Phi$

$$
\begin{aligned}
\tilde{H}[T] \tilde{\Psi}[\Phi] & :=\int_{\Sigma} d x\left\langle\Phi\left|\hat{P}\left(a_{1} \hat{m}_{1}+a_{2} \hat{m}_{2}+a_{3} \hat{m}_{3}\right)\right| \Psi\right\rangle \\
& =\left(a_{1} n_{1}(\Psi)+a_{2} n_{2}(\Psi)+a_{3} n_{3}(\Psi)\right) \delta_{\Psi, \Phi},
\end{aligned}
$$

where we have used equation (58) and the orthogonality of spin-network states in the kinematical inner product. The coefficients $n_{1}, n_{2}, n_{3}$ count the number of times that the operators $\hat{m}_{1}, \hat{m}_{2}$ and $\hat{m}_{3}$ respectively can act on the state $\Psi$. But since these coefficients are state dependent and the coefficients $a, b, c$ are not, the action of $\tilde{H}[T]$ cannot be made to vanish on all physical states.

So from our perspective it seems clear that the difficulties in generalising the definition of the loop quantum gravity Hamiltonian stems from identifying the move operators with Hamiltonian actions and not evolutions. This is in line with views expressed by Markopoulou [14, where evolution of spin networks is described in terms of the Pachner moves listed above. In so far as these models should reproduce quantum gravity in $2+1$ dimensions our work gives them a precise motivation in terms of canonical loop quantum gravity and shows that the amplitudes for these transitions should be given by the 6 -J symbols as described above.

The problem we face is to find a Hamiltonian which can reproduce the elementary moves when exponentiated. This seems a difficult task. One possibility is that it would be possible to quantise the action generated by the classical Hamiltonian directly, in the same way that it is only possible to solve the diffeomorphism constraint by quantising the action generated by it. 
Another option that is currently being explored is to make use of the close connection to the classical formulation of $2+1$ gravity in terms of 't Hoofts lattice model [27]. Here states are described by trivalent graphs with labellings corresponding to edge lengths. It can be shown that Hamiltonian evolution increases and decreases the edge lengths. Crucially, edge lengths can evolve to zero at which point a change in the topology of the graph can occur. These possible changes have been tabulated in [28] and, in the absence of matter, they turn out to be precisely the three elementary evolution moves. Hence it is possible that Hamiltonian action should act on edges by increasing and decreasing the values of the spins by adding loops of spin 1 to all closed loops in the graph of a given spin-network.

4.6. Generalisations and outlook. Our results hold for $B F$ theories in any dimension, which are all topological field theories. These models are also expressed in terms of a single triangulation, where the $6-\mathrm{J}$ symbols are replaced by their higher dimensional analogues.

The situation looks more complicated for quantum gravity since in space-time dimension higher than 3 the theory has an infinite number of degrees of freedom, and is no longer equivalent to $B F$ theory and no longer topological. However there still exist spin-foam models that attempt to define a path integral for the theory both for Lorentzian and Euclidean signatures (see [17 for a review). In this case the transition amplitudes are no longer triangulation independent and we need to include the sum over triangulations to restore covariance. However, our results have shown that the sum over triangulations should be restricted to triangulations with a slicing structure. Such restricted sums have been studied in the context of Lorentzian dynamical triangulation models [2] and there is mounting evidence that they have better behaviour than sums over generic triangulations.

In addition, as soon as spin-foam models are no longer topological, taking a continuum limit will become important. Our work indicates that in loop quantum gravity and related spin-foam models this limit involves considering large spins while taking the Immirzi parameter $\gamma$ to 0 . This has been suggested independently by Bojowald in the context of cosmological models 国. In addition, the study of the geometric operators area, volume and angle around a vertex of a spin-network carried out by Major and Seifert 13 has revealed that in order to approximate continuum geometries resembling what we observe, we need to consider a large spin limit.

We note also that for non-topological theories we can no longer argue that the elementary moves leave physical states invariant. Presumably again this is linked to the need to take the continuum limit in defining the evolution operators. The physical consequences of this deserves to be investigated in more detail.

\section{ACKNOWLEDGEMENTS}

We are grateful to Jan Ambjørn for continuing support throughout this work, especially for the idea on how to derive the Hamiltonian constraint in $(1+1)$ dynamical triangulations. We would also like to thank Marcus Gaul, B. Durhuus and Carlo Rovelli for helpful discussions. This work was supported by the EU-network on "Discrete Random Geometries" grant HPRN-CT-1999-00161. 


\section{APPENDIX}

We show that if a triangulation is generated by the action of an ordered product $\hat{U}_{l, a}\left(x_{1}\right) \cdots \hat{U}_{l, a}\left(x_{s}\right)$ acting on an auxiliary state, then this is the unique sequence of operators that will produce this triangulation, where $\hat{U}_{l, a}(x)$ is defined in equation (22). We proceed by induction over the number of triangles in a triangulation.

Clearly the addition of one triangle to a boundary triangulation corresponds to a unique action of on $\hat{U}_{l, a}(x)$. The position $x$ is given by the co-ordinate of the node on the right edge of the triangle glued to the boundary.

Let us assume that any triangulation with $n$ triangles has a unique sequence of evolution operators corresponding to it. Now let us consider a triangulation with $n+1$ triangles, which can be described by at least one sequence of operators. This triangulation will have a set of triangles that is attached to the boundary. This set inherits an ordering from the order of the coordinates of the nodes on the edges that the triangles are glued to. Hence, the triangulation will contain a unique triangle, which is defined by the fact that it is attached to a boundary edge with the largest value of the node coordinate. Since the products of evolution operators in the expansion of $\hat{P}_{l, a}$ are ordered this triangle has to be constructed first. Hence the first operator in any sequence of operators corresponding to our triangulation is fixed and unique.

But now we need to add only $n$ more triangles to obtain our triangulation. By assumption there is only one sequence of operators that will describe this triangulation with $n$ triangles.

Hence we conclude that every triangulation with $n+1$ triangles has a unique corresponding product of operators $\hat{U}_{l, a}\left(x_{1}\right) \cdots \hat{U}_{l, a}\left(x_{n+1}\right)$. qed.

\section{REFERENCES}

[1] J. Ambjorn, J. Jurkiewicz, and R. Loll. Lorentzian and euclidean quantum gravity: Analytical and numerical results. 1999, hep-th/0001124.

[2] J. Ambjorn, J. Jurkiewicz, and R. Loll. Dynamically triangulating lorentzian quantum gravity. Nucl. Phys., B610:347-382, 2001, hep-th/0105267.

[3] John C. Baez. An introduction to spin foam models of bf theory and quantum gravity. Lect. Notes Phys., 543:25-94, 2000, gr-qc/9905087.

[4] Martin Bojowald. The semiclassical limit of loop quantum cosmology. Class. Quant. Grav., 18:L109-L116, 2001, gr-qc/0105113.

[5] Steven Carlip. Quantum gravity in 2+1 dimensions. Cambridge University Press, 1998.

[6] B. Durhuus and C. W.H. Lee. A string bit hamiltonian approach to two-dimensional quantum gravity. 2001, arXiv:hep-th/0108149.

[7] Marcus Gaul and Carlo Rovelli. A generalized hamiltonian constraint operator in loop quantum gravity and its simplest euclidean matrix elements. 2000, gr-qc/0011106.

[8] D. Giulini. Group averaging and refined algebraic quantization. Nucl. Phys. Proc. Suppl., $88: 385,2000$, gr-qc/0003040.

[9] Andres Gomberoff. On group averaging for non-compact groups. 2000, hep-th/0012040.

[10] Jonathan J. Halliwell and Miguel E. Ortiz. Sum over histories origin of the composition laws of relativistic quantum mechanics. Phys. Rev., D48:748, 1993, gr-qc/9211004.

[11] Giorgio Immirzi. Quantum gravity and regge calculus. Nucl. Phys. Proc. Suppl., 57:65, 1997, gr-qc/9701052.

[12] John R. Klauder. Quantization of constrained systems. Lect. Notes Phys., 572:143-182, 2001, hep-th/0003297.

[13] Seth A. Major and Michael D. Seifert. Modelling space with an atom of quantum geometry. 2001, gr-qc/0109056.

[14] Fotini Markopoulou. Dual formulation of spin network evolution. 1997, gr-qc/9704013. 
[15] Donald Marolf. Group averaging and refined algebraic quantization: Where are we now? 2000, gr-qc/0011112.

[16] Hirosi Ooguri. Partition functions and topology changing amplitudes in the 3-d lattice gravity of ponzano and regge. Nucl. Phys., B382:276-304, 1992, hep-th/9112072.

[17] Daniele Oriti. Spacetime geometry from algebra: Spin foam models for non- perturbative quantum gravity. 2001, gr-qc/0106091.

[18] G. Ponzano and T. Regge. Semiclassical limit of racah coefficients. In F. Bloch, editor, Spectroscopic and Group Theoretical Methods in Physics, New York, 1968. NorthHolland.

[19] Michael P. Reisenberger. World sheet formulations of gauge theories and gravity. 1994, arXiv:gr-qc/9412035.

[20] Michael P Reisenberger and Carlo Rovelli. *sum over surfaces* form of loop quantum gravity. Phys. Rev., D56:3490-3508, 1997, gr-qc/9612035.

[21] Michael P. Reisenberger and Carlo Rovelli. Spacetime as a feynman diagram: The connection formulation. Class. Quant. Grav., 18:121-140, 2001, gr-qc/0002095.

[22] Carlo Rovelli. The basis of the ponzano-regge-turaev-viro-ooguri quantum gravity model in the loop representation basis. Phys. Rev., D48:2702-2707, 1993, hep-th/9304164

[23] Carlo Rovelli. The projector on physical states in loop quantum gravity. Phys. Rev., D59:104015, 1999, gr-qc/9806121.

[24] Carlo Rovelli and Lee Smolin. Spin networks and quantum gravity. Phys. Rev., D52:57435759, 1995, gr-qc/9505006.

[25] Carlo Rovelli and Thomas Thiemann. The immirzi parameter in quantum general relativity. Phys. Rev., D57:1009-1014, 1998, gr-qc/9705059.

[26] Lee Smolin. The classical limit and the form of the hamiltonian constraint in nonperturbative quantum general relativity. 1996, gr-qc/9609034.

[27] G. 't Hooft. Classical n particle cosmology in (2+1)-dimensions. In *'t Hooft, G. (ed.): Under the spell of the gauge principle* 606-618, and Class. Quantum Grav. 10 (1993) Suppl., pp 79-91.

[28] G. 't Hooft. The evolution of gravitating point particles in (2+1)- dimensions. Class. Quant. Grav., 10:1023-1038, 1993.

[29] Claudio Teitelboim. Quantum mechanics of the gravitational field. Phys. Rev., D25:3159, 1982.

[30] Claudio Teitelboim. Causality versus gauge invariance in quantum gravity and supergravity. Phys. Rev. Lett., 50:705, 1983.

[31] Claudio Teitelboim. The proper time gauge in quantum theory of gravitation. Phys. Rev., D28:297, 1983.

[32] T. Thiemann. Quantum spin dynamics (qsd). Class. Quant. Grav., 15:839-873, 1998, gr-qc/9606089.

[33] V. G. Turaev and O. Y. Viro. State sum invariants of 3 manifolds and quantum $6 \mathrm{j}$ symbols. Topology, 31:865-902, 1992.

[34] Edward Witten. (2+1)-dimensional gravity as an exactly soluble system. Nucl. Phys., B311:46, 1988.

Niels Bohr Institute, Blegdamsvej 17, DK-2100 Copenhagen $\varnothing$

E-mail address: arnsdorf@nbi.dk 